\title{
Simulation for Ruin Probabilities in Insurance with Sequence Markov Dependence Random Variables
}

\author{
Quang Phung Duy ${ }^{1, *}$, Thinh Nguyen Huu ${ }^{1}$, Chien Doan Quyet ${ }^{2}$ \\ ${ }^{1}$ Mathematics Department, Foreign Trade University, Hanoi, Viet Nam \\ ${ }^{2}$ Statistics \& Actuarial Science, Soongsil University, Seoul, South Korea
}

Received June 30, 2021; Revised August 1, 2021; Accepted September 21, 2021

\section{Cite This Paper in the following Citation Styles}

(a): [1] Quang Phung Duy, Thinh Nguyen Huu, Chien Doan Quyet, "Simulation for Ruin Probabilities in Insurance with Sequence Markov Dependence Random Variables, "Universal Journal of Accounting and Finance, Vol. 9, No. 5, pp. 927 - 934, 2021. DOI: 10.13189/ujaf.2021.090504.

(b): Quang Phung Duy, Thinh Nguyen Huu, Chien Doan Quyet (2021). Simulation for Ruin Probabilities in Insurance with Sequence Markov Dependence Random Variables. Universal Journal of Accounting and Finance, 9(5), 927 - 934. DOI: 10.13189/ujaf.2021.090504.

Copyright $\odot 2021$ by authors, all rights reserved. Authors agree that this article remains permanently open access under the terms of the Creative Commons Attribution License 4.0 International License

\begin{abstract}
The aim of this paper is to calculate ruin probabilities using Monte Carlo method for two models: i) classical risk model with claim amounts are homogeneous Markov chains; ii) generalized risk models with premiums amounts, claim amounts are homogeneous Markov chains. The sequence of random variables in the article is considered as a series of Markov dependent random variables. The main results of this paper are Lemma 3.1, Lemma 3.2 and Lemma 3.3, which have built mathematical formulas for the simulation of the probability of insurance models considered in this paper. From those lemmas, we build algorithms to simulate ruin probability for insurance models considered in this paper. From these algorithms, we build numerical results illustrating the problems posed in the paper. These results all show that when the initial capital increases, the ruin probability will decrease, and when the time increases, the ruin probability will increase. This result is consistent with the theory of the risk problem in insurance.
\end{abstract}

Keywords Ruin Probability, Homogeneous Markov Chain, Monte Carlo Method

JEL Codes: 62P05, 60G40, 12E05.

\section{Introduction}

In risk theory, the premiums amount $\mathrm{U}(\mathrm{t})$ at time $\mathrm{t}$ :
$\mathrm{U}(\mathrm{t})=\mathrm{u}+\mathrm{rt}-\sum_{\mathrm{i}=1}^{\mathrm{N}_{\mathrm{t}}} \mathrm{X}_{\mathrm{i}}$, where $\mathrm{u}>0$ is the initial capital of that company, and $r$ is the premium rate per a unit of time. The number of claim amounts to time $t, \mathrm{~N}_{\mathrm{t}}$ is the pure Poisson process with intensity $\mu$ and claim amount series $\left\{\mathrm{X}_{\mathrm{i}}\right\}$ is a series of independent random variables having the same distribution as the probability distribution function $F$, which have finite mean $\mu$. The ruin probability with finite time $t$, denoted $\psi(u, t)$, is defined by:

$$
\psi(\mathrm{u}, \mathrm{t})=\mathrm{P}\{\exists \tau \leq \mathrm{t}: \mathrm{U}(\tau)<0\}
$$

Ruin probability with infinite time, denoted $\psi(\mathrm{u})$, is defined by:

$$
\psi(\mathrm{u})=\psi(\mathrm{u},+\infty)=\lim _{\mathrm{t} \rightarrow+\infty} \psi(\mathrm{u}, \mathrm{t})
$$

If there exists a number $\mathrm{R}>0$ satisfying

$$
\int_{0}^{+\infty} \mathrm{e}^{\mathrm{Rx}}(1-\mathrm{F}(\mathrm{x})) \mathrm{dx}=\frac{\mathrm{r}}{\mu}
$$

then with every $\mathrm{u} \geq 0$ we have $\psi(\mathrm{u}) \leq \mathrm{e}^{-\mathrm{Ru}}$. If $\int_{0}^{+\infty} \mathrm{e}^{\mathrm{Rx}}(1-\mathrm{F}(\mathrm{x})) \mathrm{dx}<+\infty$ then

$$
\lim _{u \rightarrow+\infty} e^{R u} \psi(u)=C
$$

where $\mathrm{C}$ is a constant. Equation (1.3) is called aproximate 
Cramer - Lundberg and $\mathrm{R}$ is called exponential constant Lundberg. (see H. U. Gerber [3] and Grandell [4]). For these dependency structure models, it would often be very hard to calculate the approximation of exponential constant R (see Phung Duy Quang [5], [6]). In [1], authors considered the numerical solution to one type of integro-differential equation by a probability method based on the fundamental martingale of mixed Gaussian processes. As an application, we try to simulate the estimation of ruin probability with an unknown parameter driven not by the classical Lévy process, but by the mixed fractional Brownian motion. In [2], authors studied based on a discrete version of the Pollaczeck-Khinchine formula, a general method of calculating the ultimate ruin probability in the Gerber-Dickson risk model is provided when claims follow a negative binomial mixture distribution. The result is then extended for claims with a mixed Poisson distribution. The formula obtained allows for some approximation procedures.

Analytical results and numerical results are often unknown. Simulation Monte Carlo methods can provide tools for calculating approximately probabilities $\psi(\mathrm{u}, \mathrm{t})$.

The aim of this paper is to approximately calculate ruin probability $\psi(\mathrm{u}, \mathrm{t})$ in two cases using Monte Carlo simulation method: i) the claim amounts is an homogeneous Markov chain in classical model; ii) premiums amounts, claim amounts are homogeneous Markov chains in the general model does not have effects of interests.

In the second section of this paper, authors will introduce the classical model, the general model that has no effect of interest rates with a series of Markov dependent random variables. In the third section of this paper, authors will introduce simulation algorithms to calculate ruin probability in the models introduced in the second section of this paper. In the fourth section of this paper, authors will introduce simulation results with different homogeneous Markov chain dependent models. Finally, the fifth section concludes the paper.

\section{Insurance Model with Homogeneous Markov Chain Dependent Random Variables}

\subsection{Classical Risk Model}

In the classical risk model, we assume that the capital of the insurance company at time $t$ is:

$$
U(t)=u+r t-S_{t}=u+r t-\sum_{k=1}^{N_{t}} X_{k}
$$

Where $u$ is the initial capital, $r$ is the cost of credit, $\mathrm{X}_{\mathrm{t}}$ is the claim amount at time $t ; \mathrm{N}_{\mathrm{t}}$ is the number of claims up to time $\mathrm{t}\left(\mathrm{N}_{\mathrm{t}}\right.$ is the pure Poisson process with intensity $\mu$, the interval between two claims, which is independent and co-distributed, following an exponential distribution with parameter $\mu$, expectation $\left.\frac{1}{\mu}\right) ; X_{t}$ is a homogeneous Markov chain independent of $\mathrm{N}_{\mathrm{t}}$; the total claim amounts up to time $t$ is $S_{t}=\sum_{k=1}^{N_{t}} X_{k}$.

Ruin probability to time $\mathrm{t}$ is determined by:

$$
\psi(\mathrm{u}, \mathrm{t})=\mathrm{P}(\exists \tau \leq \mathrm{t}: \mathrm{U}(\tau)<0)
$$

\subsection{The General Risk Model where There Is No Interest Rate Effect}

In the general risk model where there is no interest rate effect, we assume that the capital of the insurance company at time $t$ is:

$$
\mathrm{U}(\mathrm{t})=\mathrm{u}+\sum_{\mathrm{i}=1}^{\mathrm{N}_{t}^{1}} \mathrm{X}_{\mathrm{i}}-\sum_{\mathrm{j}=1}^{\mathrm{N}_{\mathrm{t}}^{2}} \mathrm{Y}_{\mathrm{j}}
$$

Where $\mathrm{u}$ is the initial capital; the series of premium amounts $X_{1}, X_{2}, \ldots, X_{n}$ depends on homogeneous Markov chain; series of claim amounts $Y_{1}, Y_{2}, \ldots, Y_{n}$ depends on homogeneous Markov chain $\left(\mathrm{X}_{\mathrm{t}}\right.$ is independent on $\left.\mathrm{Y}_{\mathrm{t}}\right)$; $\mathrm{N}_{\mathrm{t}}^{1}$ is the number of premium amounts up to time $t$ with $\mathrm{N}_{\mathrm{t}}^{1}$ is the pure Poisson process with intensity $\mu_{1}>0$ (the time interval between two premium is independent and co-distributed, following an exponential distribution with parameter $\mu_{1}$, the expectation is $\left.\frac{1}{\mu_{1}}\right), X_{t}$ is independent on $\mathrm{N}_{\mathrm{t}}^{1} ; \mathrm{N}_{\mathrm{t}}^{2}$ is the number of claim amounts to time $\mathrm{t}$ with $\mathrm{N}_{\mathrm{t}}^{2}$ is the pure Poisson process with intensity $\mu_{2}>0$ (the time interval between two claims is independent and co-distributed, following an exponential distribution with parameter $\mu_{2}$, the expectation is $\left.\frac{1}{\mu_{2}}\right), Y_{\mathrm{t}}$ is independent on $\mathrm{N}_{\mathrm{t}}^{2} ; \mathrm{N}_{\mathrm{t}}^{1}$ is independent on $\mathrm{N}_{\mathrm{t}}^{2}$.

The ruin probability to time $t$ is determined by:

$$
\psi(\mathrm{u}, \mathrm{t})=\mathrm{P}(\exists \tau \leq \mathrm{t}: \mathrm{U}(\tau)<0)
$$

\section{The Monte Carlo Simulation Method Approximates the Ruin Probabilities in the Insurance Model}

\subsection{The Algorithm to Simulate A Homogeneous Markov Chain}

We assume that $X_{n}$ are defined on the probability space $(\Omega, A, P) .\left\{X_{n}\right\}_{n \geq 0}$ is an homogeneous Markov chain, such that for any $n$ the values of $X_{n}$ are taken from a set of 
non - negative numbers $G=\left\{x_{1}, x_{2}, \ldots, x_{n}, \ldots\right\}$ with $x_{o}=x_{i}$ and

$\mathrm{p}_{\mathrm{ij}}=\mathrm{P}\left[\omega \in \Omega: \mathrm{X}_{\mathrm{n}+1}(\omega)=\mathrm{x}_{\mathrm{j}} \mid \mathrm{X}_{\mathrm{n}}(\omega)=\mathrm{x}_{\mathrm{i}}\right]\left(\mathrm{n} \in \mathrm{N}, \mathrm{x}_{\mathrm{i}} \in \mathrm{G}, \mathrm{x}_{\mathrm{j}} \in \mathrm{G}\right)$, where $0 \leq \mathrm{p}_{\mathrm{ij}} \leq 1, \sum_{\mathrm{j}=1}^{+\infty} \mathrm{p}_{\mathrm{ij}}=1$.

Let $\mathrm{Y}_{\mathrm{i}}$ denote a generic distributed as the $\mathrm{i}^{\text {th }}$ row of the matrix, that is,

$$
P\left(Y_{i}=y_{j}\right)=p_{i j} ; y_{j} \in G
$$

Let us assume we use inversion to generate such a $Y_{i}$. Generate $U$ with $U$ is uniformly distributed. Let $Y_{i}=x_{1}$, if $\mathrm{U} \leq \mathrm{p}_{\mathrm{i} 0} ; \mathrm{Y}_{\mathrm{i}}=\mathrm{x}_{2}$, if $\mathrm{p}_{\mathrm{io}}<\mathrm{U} \leq \mathrm{p}_{\mathrm{i} 0}+\mathrm{p}_{\mathrm{i}, 1}$; and in general $\mathrm{Y}_{\mathrm{i}}=\mathrm{x}_{\mathrm{j}}$, if $\sum_{\mathrm{k}=0}^{\mathrm{j}-1} \mathrm{p}_{\mathrm{iik}}<\mathrm{U} \leq \sum_{\mathrm{k}=0}^{\mathrm{j}} \mathrm{p}_{\mathrm{ik}}$. In the following algorithm, whenever we say "generate a $\mathrm{Y}_{\mathrm{i}}$ ", we mean doing so using this inverse transform method using uniform distribution.

We will build a homogeneous Markov chain simulation algorithm.

\section{Algorithm 3.1.}

Input: $\mathrm{G}=\left\{\mathrm{x}_{1}, \mathrm{x}_{2}, \ldots, \mathrm{x}_{\mathrm{n}}, \ldots\right\} ; \mathrm{P}=\left[\mathrm{p}_{\mathrm{ij}}\right]_{\mathrm{n} \times \mathrm{n}}$ is transition matrix.

Output: $X_{n}: X_{n}$ is a homogeneous Markov chain, $X_{n}$ are taken from $G$

\section{Steps of the algorithm:}

Step 1. Choose an initial value, $X_{o}=x_{i_{o}}$. Set $n=1$

Step 2. Generate $Y_{i_{o}}$, and set $X_{1}=Y_{i_{o}}$.

Step 3. If $n<N$, then set $i=X_{n}$, generate $Y_{i}$, set $n=n$ +1 and set $X_{n}=Y_{i}$; otherwise stop.

Step 4. Go back to Step3.

\subsection{The Algorithm to Simulate Ruin Probability for the Model (2.1)}

We see model (2.1) with a series of random variables $\left\{\mathrm{X}_{\mathrm{k}}\right\}_{\mathrm{k}=1}^{\mathrm{n}}$ depends on homogeneous Markov chain. If we call $\left\{\tau_{\mathrm{i}}\right\}_{\mathrm{i} \geq 1}$ as a series of independent random variables, with the same distribution $\mathrm{E}\{\mu\}$ (indicates the time between claims $\left.\left\{T_{i}\right\}_{i=1}^{N_{t}}\right)$, then we have:

$$
\begin{aligned}
& \mathrm{N}_{\mathrm{t}}:=\max \left\{\mathrm{k}: \sum_{\mathrm{i}=1}^{\mathrm{k}} \tau_{\mathrm{i}}:=\mathrm{T}_{\mathrm{k}} \leq \mathrm{t}\right\} ; \\
& \tau_{\mathrm{o}}=\mathrm{T}_{\mathrm{o}}=0, \tau_{\mathrm{i}}=-\frac{\ln \mathrm{v}_{\mathrm{i}}}{\mu} ; \mathrm{v}_{\mathrm{i}} \square \mathrm{U}(0 ; 1)(\mathrm{i} \geq 1)
\end{aligned}
$$

In which, random numbers $v_{i}(i \geq 1)$ is independent.

We, now, consider event $\mathrm{A}(\mathrm{t})$ (up to time $\mathrm{t}$ ) of the problem (2.1):

$$
\psi(\mathrm{u}, \mathrm{t})=\mathrm{P}\{\mathrm{A}(\mathrm{t})\}, \mathrm{A}(\mathrm{t}):=\{\exists \mathrm{s} \leq \mathrm{t}: \mathrm{U}(\mathrm{s})<0\}
$$

The basis for simulating event $\mathrm{A}(\mathrm{t})$ is the following proposition:

Lemma 3.1. If we let $\psi(\mathrm{u}, \mathrm{t})=\mathrm{A}(\mathrm{t}):=\{\exists \mathrm{s} \leq \mathrm{t}: \mathrm{U}(\mathrm{s})<0\}$ then $\mathrm{A}(\mathrm{t})=\bigcup_{\mathrm{i}=0}^{\mathrm{N}_{\mathrm{t}}}\left\{\mathrm{U}\left(\mathrm{T}_{\mathrm{i}}\right)<0\right\}$.

\section{Prove:}

Without losing generality, we assume $\mathrm{N}_{t} \geq 1$, we set

$$
<T_{j-1}, T_{j}>:= \begin{cases}\left(0, T_{1}\right) & \text { if } j=1, \\ {\left[T_{j-1}, T_{j}\right)} & \text { if } j=2 \div N_{t}, \\ {\left[T_{N_{t}}, t\right]} & \text { if } j=N_{t}+1 .\end{cases}
$$

Then from (3.1) we have:

$$
\bigcup_{\mathrm{j}=1}^{\mathrm{N}_{\mathrm{t}}+1}<\mathrm{T}_{\mathrm{j}-1}, \mathrm{~T}_{\mathrm{j}}>=(0, \mathrm{t}],<\mathrm{T}_{\mathrm{j}-1}, \mathrm{~T}_{\mathrm{j}}>\cap<\mathrm{T}_{\mathrm{i}-1}, \mathrm{~T}_{\mathrm{i}}>=\phi(\forall \mathrm{i} \neq \mathrm{j})
$$

To point out that:

$$
\mathrm{U}(\mathrm{s})=\mathrm{U}\left(\mathrm{T}_{\mathrm{j}-1}\right)\left(\forall \mathrm{s} \in<\mathrm{T}_{\mathrm{j}-1}, \mathrm{~T}_{\mathrm{j}}>, \mathrm{j}=1 \div \mathrm{N}_{\mathrm{t}}+1\right),
$$

And $\mathrm{U}(\mathrm{s})=\mathrm{U}\left(\mathrm{T}_{\mathrm{o}}\right)=\mathrm{u}>0, \quad \forall \mathrm{s} \in<\mathrm{T}_{\mathrm{o}}, \mathrm{T}_{1}>$.

Let $\mathrm{A}_{\mathrm{j}}(\mathrm{t}):=\left\{\exists \mathrm{s} \in<\mathrm{T}_{\mathrm{j}-1}, \mathrm{~T}_{\mathrm{j}}>\mathrm{U}(\mathrm{s})<0\right\}\left(\forall \mathrm{j}=1 \div \mathrm{N}_{\mathrm{t}}+1\right)$.

Then

$$
A(t)=\bigcup_{j=1}^{N_{t}+1} A_{j}(t)=\bigcup_{j=2}^{N_{t}+1} A_{j}(t)
$$

because $\mathrm{A}_{1}(\mathrm{t}):=\left\{\exists \mathrm{s} \in<\mathrm{T}_{\mathrm{o}}, \mathrm{T}_{1}>: \mathrm{U}(\mathrm{s})<0\right\}=\phi$.

On the other hand:

$$
\begin{aligned}
& \left\{\mathrm{U}\left(\mathrm{T}_{\mathrm{j}-1}\right)<0\right\} \subset \mathrm{A}_{\mathrm{j}}(\mathrm{t}) \subset\left\{\mathrm{U}\left(\mathrm{T}_{\mathrm{j}-1}\right)<0\right\} \\
& \Rightarrow \mathrm{A}_{\mathrm{j}}(\mathrm{t})=\left\{\mathrm{U}\left(\mathrm{T}_{\mathrm{j}-1}\right)<0, \forall \mathrm{j}=2 \div \mathrm{N}^{2}(\mathrm{t})+1\right\}
\end{aligned}
$$

Then

$$
A(t)=\bigcup_{j=2}^{N_{t}+1}\left\{U\left(T_{j-1}\right)<0\right\}=\bigcup_{j=1}^{N_{t}}\left\{U\left(T_{j}\right)<0\right\}
$$

From Lemma 3.1, the ruin probability at (2.4) is estimated as:

$$
\begin{aligned}
& \psi(\mathrm{u}, \mathrm{t})=\mathrm{P}\{\mathrm{A}(\mathrm{t})\} \approx \frac{\mathrm{M}}{\mathrm{N}} ; \mathrm{A}(\mathrm{t}) \\
& :=\{\exists \mathrm{s} \leq \mathrm{t}: \mathrm{U}(\mathrm{s})<0\}=\bigcup_{\mathrm{i}=0}^{\mathrm{N}_{\mathrm{t}}}\left\{\mathrm{U}\left(\mathrm{T}_{\mathrm{i}}\right)<0\right\}
\end{aligned}
$$

Where $M$ is the number of occurrences of event $A(t)$ in $\mathrm{N}$ simulations and $\mathrm{M}$ is determined by the following algorithm.

\section{Algorithm 3.2.}

Input: initial capital $\mathrm{u}$, cost rate $\mathrm{r}$, time $\mathrm{t}$, the number of simulations $N$, parameter $\mu, G=\left\{x_{1}, x_{2}, \ldots, x_{n}, \ldots\right\} ; P=$ $\left[\mathrm{p}_{\mathrm{ij}}\right]_{\mathrm{n} \times \mathrm{n}}$ is transition matrix

Output: Risk probability $\psi(\mathrm{u}, \mathrm{t})$ 


\section{Steps of the algorithm:}

First of all, assign $M=0, T_{o}=0, U\left(T_{0}\right):=u$.

Step A. (in the $\mathrm{n}=\overline{1, \mathrm{~N}}$ ). With each $\mathrm{i}=1,2, \ldots$ We do it as follows:

A1. Simulate the time to claim: $T_{i}=T_{i-1}+\tau_{i}$ with $\tau_{i}$ created according to the formula (3.1) and check inequality:

$$
\mathrm{T}_{\mathrm{i}} \leq \mathrm{t}
$$

- If (3.3a) is false: terminate the $\mathrm{n}^{\text {th }}$ simulation of event $\mathrm{A}(\mathrm{t})$.

- If (3.3a) is true: move to step A2.

A2. Simulation of claim value $X_{i}$ according to algorithm 3.1 to calculate (see (2.1)):

$$
\mathrm{U}\left(\mathrm{T}_{\mathrm{i}}\right)=\mathrm{U}\left(\mathrm{T}_{\mathrm{i}-1}\right)+\mathrm{r}\left(\mathrm{T}_{\mathrm{i}}-\mathrm{T}_{\mathrm{i}-1}\right)-\mathrm{X}_{\mathrm{i}}
$$

and check inequality:

$$
\mathrm{U}\left(\mathrm{T}_{\mathrm{i}}\right) \geq 0
$$

- If (3.3b) is false: terminate the simulation at the $\mathrm{n}^{\text {th }}$ time of event $\mathrm{A}(\mathrm{t})$ and assign $\mathrm{M}:=\mathrm{M}+1$

- If (3.3a) is true: Move back to step A1 with $\mathrm{i}:=\mathrm{i}+1$

Notice that: the loop will stop when $\mathrm{i}=\mathrm{N}_{\mathrm{t}}(\mathrm{xem}$ (3.1)) and finish the $\mathrm{n}^{\text {th }}$ simulation of event $\mathrm{A}(\mathrm{t})$.

Step B. After simulating $\mathrm{N}$ times event $\mathrm{A}(\mathrm{t})$ (repeat $\mathrm{N}$ times step A, approximately calculate the probability of risk: $\Psi(\mathrm{u}, \mathrm{t})=\frac{\mathrm{M}}{\mathrm{N}}$.

\subsection{Algorithm to Simulate Ruin Probability for the Model (2.2)}

To describe the method, we consider the model (2.2) with the assumption that: series of amounts $\left\{X_{i}\right\}_{i \geq 1}$ and the series of the claim amount $\left\{\mathrm{Y}_{\mathrm{j}}\right\}_{\mathrm{j} \geq 1}$ are homogeneous Markov chains.

Let $\mathrm{N}_{\mathrm{s}}^{\mathrm{k}} \equiv \mathrm{N}^{\mathrm{k}}(\mathrm{s})(\mathrm{k}=\overline{1,2})$ the Poisson process with intensity $\mu_{\mathrm{k}}$, represents the number of receiving times (when $\mathrm{k}=1$ ) and the number of payments (when $\mathrm{k}=2$ ) in period $(0, \mathrm{~s}]$. Let $\mathrm{T}_{\mathrm{i}}^{\mathrm{k}}, \mathrm{T}_{\mathrm{i}}^{\mathrm{k}}$ the receiving time $($ when = 1) and claim payment (when $\mathrm{k}=2$ ) in the ith time. Then from (3.1), we have:

$$
\begin{gathered}
\mathrm{N}_{\mathrm{s}}^{\mathrm{k}} \equiv \mathrm{N}^{\mathrm{k}}(\mathrm{s}):=\max \left\{\mathrm{i}: \sum_{\mathrm{j}=0}^{\mathrm{i}} \tau_{\mathrm{j}}^{\mathrm{k}}:=\mathrm{T}_{\mathrm{i}}^{\mathrm{k}} \leq \mathrm{s}\right\} ; \\
\tau_{\mathrm{o}}^{\mathrm{k}}=\mathrm{T}_{\mathrm{o}}^{\mathrm{k}}=0(\mathrm{k}=\overline{1,2}) \\
\tau_{\mathrm{j}}^{\mathrm{k}}:=\frac{-\ln \mathrm{v}_{\mathrm{j}}^{\mathrm{k}}}{\mu_{\mathrm{k}}}, \mathrm{v}_{\mathrm{j}}^{\mathrm{k}} \sim \mathrm{U}(0,1)(\forall \mathrm{j} \geq 1, \mathrm{k}=\overline{1,2})
\end{gathered}
$$

In which, for each $\mathrm{k}=\overline{1,2}, \mathrm{v}_{\mathrm{j}}^{\mathrm{k}}(\mathrm{j} \geq 1)$ are independent random numbers. Then we can determine capital process $U\left(T_{j}^{2}\right)(j \geq 1)$ of the insurance company at the time of claim $\mathrm{T}_{\mathrm{j}}^{2}$, through the following proposition:

Lemma 3.2. 1) With the above assumptions, if $N^{2}(t)>0$ and $N^{1}\left(T_{j-1}^{2}\right)<N^{2}\left(T_{j}^{2}\right)(\forall j \geq 1)$ then it is almost sure (a.s) that:

$$
\left.\begin{array}{l}
0<\mathrm{T}_{1}^{1}<\ldots<\mathrm{T}_{\mathrm{N}^{1}\left(\mathrm{~T}_{1}^{2}\right)}^{1} \leq \mathrm{T}_{1}^{2}<\mathrm{T}_{\mathrm{N}^{1}\left(\mathrm{~T}_{1}^{2}\right)+1}^{1} \\
<\ldots<\mathrm{T}_{\mathrm{N}^{1}\left(\mathrm{~T}_{\mathrm{j}-1}^{2}\right)}^{1} \leq \mathrm{T}_{\mathrm{j}-1}^{2}<\mathrm{T}_{\mathrm{N}^{1}\left(\mathrm{~T}_{\mathrm{j}-1}^{2}\right)+1}^{1} \\
<\ldots<\mathrm{T}_{\mathrm{N}^{1}\left(\mathrm{~T}_{\mathrm{j}}^{2}\right)}^{1} \leq \mathrm{T}_{\mathrm{j}}^{2}<\mathrm{T}_{\mathrm{N}^{1}\left(\mathrm{~T}_{\mathrm{j}}^{2}\right)+1}^{1} \\
<\ldots<\mathrm{T}_{\mathrm{N}^{1}\left(\mathrm{~T}_{\mathrm{N}^{2}(\mathrm{t})}^{2}\right)}^{1} \leq \mathrm{T}_{\mathrm{N}^{2}(\mathrm{t})}^{2} \leq \mathrm{t}
\end{array}\right\}
$$

Then we have:

$$
\begin{aligned}
& \mathrm{U}\left(\mathrm{T}_{\mathrm{j}}^{2}\right)=\mathrm{U}\left(\mathrm{T}_{\mathrm{j}-1}^{2}\right)+\mathrm{X}\left(\mathrm{T}_{\mathrm{j}}^{2}\right)-\mathrm{Y}_{\mathrm{j}}\left(\mathrm{j}=1 \div \mathrm{N}^{2}(\mathrm{t})\right), \\
& \mathrm{U}\left(\mathrm{T}_{\mathrm{o}}^{2}\right)=\mathrm{u} ;
\end{aligned}
$$

Where

$$
X\left(T_{j}^{2}\right)= \begin{cases}0 & \text { if } N^{1}\left(T_{j-1}^{2}\right)=N^{1}\left(T_{j}^{2}\right) \\ \sum_{i=N^{1}\left(T_{j-1}^{2}\right)+1}^{N^{1}\left(T_{i}^{2}\right)} X_{i} & \text { if } N^{1}\left(T_{j-1}^{2}\right)<N^{1}\left(T_{j}^{2}\right)\end{cases}
$$

2) In case $N^{2}(t)=0$, we have:

$$
\mathrm{U}(\tau) \geq 0(\forall \tau \leq \mathrm{t})
$$

\section{Prove:}

1) From the non-trivial properties of random variables

$\tau_{\mathrm{j}}^{\mathrm{k}} \sim \mathrm{E}\left(\mu_{\mathrm{k}}\right)(\forall \mathrm{j} \geq 1)$ we infer: $\tau_{\mathrm{j}}^{\mathrm{k}}>0(\mathrm{a} . \mathrm{s}), \forall \mathrm{j} \geq 1$ then from (3.3) we have:

$$
\begin{aligned}
& 0<\mathrm{T}_{\mathrm{o}}^{2}<\mathrm{T}_{1}^{2}<\ldots<\mathrm{T}_{\mathrm{j}-1}^{2}<\mathrm{T}_{\mathrm{j}}^{2}< \\
& \ldots<\mathrm{T}_{\mathrm{N}^{2}(\mathrm{t})}^{2} \leq \mathrm{t}<\mathrm{T}_{\mathrm{N}^{2}(\mathrm{t})+1}^{2} \text { (a.s) }
\end{aligned}
$$

Therefore, when considering the definition of $\mathrm{N}^{1}(\mathrm{~s})$ (in (3.3)) with, respectively, value $s=T_{j}^{2}\left(j=1 \div \mathrm{N}^{2}(\mathrm{t})\right)$, we easily obtain (3.5).

Also, when using (3.3) with $\mathrm{k}=2$ and $\mathrm{s}=\mathrm{T}_{\mathrm{j}}^{2}$, we also have:

$$
\mathrm{T}_{\mathrm{N}^{2}\left(\mathrm{~T}_{\mathrm{j}}^{2}\right)}^{2}=\mathrm{T}_{\mathrm{j}}^{2} \Rightarrow \mathrm{N}^{2}\left(\mathrm{~T}_{\mathrm{j}}^{2}\right)=\mathrm{j}\left(\mathrm{j}=1 \div \mathrm{N}^{2}(\mathrm{t})\right) .
$$

On this basis we have the representation of $U(\tau)$ in (2.3) with $\tau=\mathrm{T}_{\mathrm{j}}^{2}$ in the form:

$$
\mathrm{U}\left(\mathrm{T}_{\mathrm{j}}^{2}\right)=\mathrm{u}+\sum_{\mathrm{i}=0}^{\mathrm{N}^{1}\left(\mathrm{~T}_{\mathrm{j}}^{2}\right)} \mathrm{X}_{\mathrm{i}}-\sum_{\mathrm{i}=0}^{\mathrm{j}} \mathrm{Y}_{\mathrm{i}}\left(1 \leq \mathrm{j} \leq \mathrm{N}^{2}(\mathrm{t})\right) \text {. }
$$

When replacing $\mathrm{j}$ in the above formula by $\mathrm{j}-1 \geq 1$, we 
have

$$
\begin{aligned}
& U\left(T_{j-1}^{2}\right)=u+\sum_{i=0}^{N^{1}\left(T_{j-1}^{2}\right)} X_{i}-\sum_{i=0}^{j-1} Y_{i} \\
& \left(2 \leq j \leq N^{2}(t)\right)
\end{aligned}
$$

For each $\mathrm{j}=2 \div \mathrm{N}^{2}(\mathrm{t})$, we rely on equations (3.6) and (3.12) to represent (3.11) in the form:

$$
U\left(T_{j}^{2}\right)= \begin{cases}U\left(T_{j-1}^{2}\right)+X\left(T_{j}^{2}\right)-Y_{j} & \text { if } N^{1}\left(T_{j-1}^{2}\right)<N^{1}\left(T_{j}^{2}\right) \\ U\left(T_{j-1}^{2}\right)-Y_{j} & \text { if } N^{1}\left(T_{j-1}^{2}\right)=N^{1}\left(T_{j}^{2}\right)\end{cases}
$$

That mean, we have (3.6) for all $\mathrm{j}=2 \div \mathrm{N}^{2}(\mathrm{t})$. Moreover, since $\mathrm{T}_{\mathrm{o}}^{2}=0, \mathrm{~N}^{1}\left(\mathrm{~T}_{\mathrm{o}}^{2}\right)=0$ (see (3.3)) so $\mathrm{U}\left(\mathrm{T}_{\mathrm{o}}^{2}\right)=\mathrm{U}(0)=\mathrm{u}$. Then since $\mathrm{X}_{\mathrm{o}}=0$ when considering (3.11) with $\mathrm{j}=1$, we can rely on (3.5) to infer:

$$
\begin{gathered}
\mathrm{U}\left(\mathrm{T}_{1}^{2}\right)=\mathrm{U}\left(\mathrm{T}_{\mathrm{o}}^{2}\right)+\sum_{\mathrm{i}=1}^{\mathrm{N}^{1}\left(\mathrm{~T}_{1}^{2}\right)} \mathrm{X}_{\mathrm{i}}-\mathrm{Y}_{1}=\mathrm{U}\left(\mathrm{T}_{\mathrm{o}}^{2}\right)+\sum_{\mathrm{i}=\mathrm{N}^{1}\left(\mathrm{~T}_{0}^{2}\right)+1}^{\mathrm{N}^{1}\left(\mathrm{~T}_{1}^{2}\right)} \mathrm{X}_{\mathrm{i}}-\mathrm{Y}_{1} \\
\text { when } \mathrm{N}^{1}\left(\mathrm{~T}_{\mathrm{o}}^{2}\right)<\mathrm{N}^{1}\left(\mathrm{~T}_{1}^{2}\right) \mathrm{a}
\end{gathered}
$$

and $\mathrm{U}\left(\mathrm{T}_{1}^{2}\right)=\mathrm{U}\left(\mathrm{T}_{0}^{2}\right)-\mathrm{Y}_{1}=\mathrm{U}\left(\mathrm{T}_{0}^{2}\right)-\mathrm{Y}_{1}$ when $\mathrm{N}^{1}\left(\mathrm{~T}_{0}^{2}\right)=\mathrm{N}^{1}\left(\mathrm{~T}_{1}^{2}\right)$. So, we get (3.6) in both the case $\mathrm{j}=1$.

2) Finally, we consider the case: $N^{2}(t)=0$. Since $0 \leq \mathrm{N}^{2}(\tau) \leq \mathrm{N}^{2}(\mathrm{t}), \forall \tau \leq \mathrm{t} \quad\left(\right.$ see $\quad(3.3), \quad \mathrm{N}^{2}(\tau)=0(\forall \tau \leq \mathrm{t})$. Then formula $\mathrm{u}_{\tau}$ in (2.3) has the form:

$$
\mathrm{U}(\tau)=\mathrm{u}+\sum_{\mathrm{i}=0}^{\mathrm{N}^{1}(\tau)} \mathrm{X}_{\mathrm{i}}-\mathrm{Y}_{\mathrm{o}}=\mathrm{u}+\sum_{\mathrm{i}=0}^{\mathrm{N}^{1}(\tau)} \mathrm{X}_{\mathrm{i}}(\forall \tau \leq \mathrm{t})
$$

Since $u>0$ and $X_{i}(i \geq 1)$ are non-negative random variables, from the above formula, we directly deduce (3.8).

Now we consider the risky event $A(t)$ (up to time $t$ ) of problem (2.2):

$$
\psi(\mathrm{u}, \mathrm{t})=\mathrm{P}\{\mathrm{A}(\mathrm{t})\}, \mathrm{A}(\mathrm{t}):=\{\exists \mathrm{s} \leq \mathrm{t}: \mathrm{U}(\mathrm{s})<0\}
$$

The basis for simulating event $\mathrm{A}(\mathrm{t})$ is the following proposition:

Lemma 3.3. In the conditions of Lemma 3.2, we have the following conclusions:

1. If $\mathrm{N}^{2}(\mathrm{t}) \geq 1$, then

$$
A(t)=B(t):=\bigcup_{j=1}^{N^{2}(t)}\left\{U\left(T_{j}^{2}\right)<0\right\}
$$

Then event $A(t)$ will not occur, if:

$$
\mathrm{U}\left(\mathrm{T}_{\mathrm{j}}^{2}\right) \geq 0\left(\forall \mathrm{j}=1 \div \mathrm{N}^{2}(\mathrm{t})\right)
$$

2. Event $A(t)$ also does not occur, if:

$$
\mathrm{N}^{2}(\mathrm{t})=0 \Leftrightarrow \tau_{1}^{2}=\frac{-\ln \mathrm{v}_{1}^{2}}{\mu_{2}}>\mathrm{t},\left(\mathrm{v}_{1}^{2} \sim \mathrm{U}(0,1)\right)
$$

\section{Prove:}

In the case of $\mathrm{N}^{2}(\mathrm{t}) \geq 1$, we assign

$$
<T_{j-1}^{2}, T_{j}^{2}>:=\left\{\begin{array}{l}
\left(0, T_{1}^{2}\right) \text { if } j=1 \\
{\left[T_{j-1}^{2}, T_{j}^{2}\right) \text { if } j:=2 \div N^{2}(t),} \\
{\left[T_{N^{2}(t)}^{2}, t\right] \text { if } j=N^{2}(t)+1 .}
\end{array}\right.
$$

Then from (3.9) we have:

$$
\begin{aligned}
& \bigcup_{\mathrm{j}=1}^{\mathrm{N}^{2}(\mathrm{t})+1}<\mathrm{T}_{\mathrm{j}-1}^{2}, \mathrm{~T}_{\mathrm{j}}^{2}>=(0, \mathrm{t}], \\
& <\mathrm{T}_{\mathrm{j}-1}^{2}, \mathrm{~T}_{\mathrm{j}}^{2}>\cap<\mathrm{T}_{\mathrm{i}-1}^{2}, \mathrm{~T}_{\mathrm{i}}^{2}>=\phi(\forall \mathrm{i} \neq \mathrm{j})
\end{aligned}
$$

To show that:

$$
\begin{aligned}
& \mathrm{U}(\mathrm{s}) \geq \mathrm{U}\left(\mathrm{T}_{\mathrm{j}-1}^{2}\right)\left(\forall \mathrm{s} \in<\mathrm{T}_{\mathrm{j}-1}^{2}, \mathrm{~T}_{\mathrm{j}}^{2}>,\right. \\
& \left.\mathrm{j}=1 \div \mathrm{N}^{2}(\mathrm{t})+1\right),
\end{aligned}
$$

Firstly, we consider the case $\mathrm{j}=1$ meaning that (see (3.17)): $0<\mathrm{s}<\mathrm{T}_{1}^{2}$. In this case, we have (see (3.3), (3.9)):

$$
\mathrm{N}^{1}(\mathrm{~s}) \geq 0,0=\mathrm{T}_{\mathrm{o}}^{2} \leq \mathrm{N}^{2}(\mathrm{~s}) \leq \mathrm{s}<\mathrm{T}_{1}^{2} \Rightarrow \mathrm{N}^{2}(\mathrm{~s})=0 .
$$

Therefore, from (2.10) we get:

$$
\begin{aligned}
& \mathrm{U}(\mathrm{s})=\mathrm{u}+\sum_{\mathrm{i}=0}^{\mathrm{N}^{1}(\mathrm{~s})} \mathrm{X}_{\mathrm{i}} \geq \mathrm{u}=\mathrm{U}(0) \\
& =\mathrm{U}\left(\mathrm{T}_{\mathrm{o}}^{2}\right)>0\left(\forall \mathrm{s} \in<\mathrm{T}_{0}^{2}, \mathrm{~T}_{1}^{2}>\right) .
\end{aligned}
$$

That means, we obtained (3.19) with $\mathrm{j}=1$. Next, we consider case $\mathrm{j}=2 \div \mathrm{N}^{2}(t)$, in which (see (3.17)): $\mathrm{T}_{\mathrm{j}-1}^{2} \leq \mathrm{s}<\mathrm{T}_{\mathrm{j}}^{2}$. Then from (3.9) and (3.3) we have: $N^{2}(s)=N^{2}\left(T_{j-1}^{2}\right)=j-1, N^{1}(s) \geq N^{1}\left(T_{j-1}^{2}\right)$. Therefore, from (2.0), (3.10) and (3.12) we deduce:

$$
\mathrm{U}(\mathrm{s}) \geq \mathrm{u}+\sum_{\mathrm{i}=0}^{\mathrm{N}^{1}\left(\mathrm{~T}_{\mathrm{j}-1}^{2}\right)} \mathrm{X}_{\mathrm{i}}-\sum_{\mathrm{i}=0}^{\mathrm{j}-1} \mathrm{Y}_{\mathrm{i}}=\mathrm{U}\left(\mathrm{T}_{\mathrm{j}-1}^{2}\right)\left(\forall \mathrm{s} \in<\mathrm{T}_{\mathrm{j}-1}^{2}, \mathrm{~T}_{\mathrm{j}}^{2}>\right) .
$$

And we obtain (3.19) with $\mathrm{j}=2 \div \mathrm{N}^{2}(\mathrm{t})$. Finally, case $\mathrm{j}=\mathrm{N}^{2}(\mathrm{t})+1$, where $\mathrm{s} \in\left[\mathrm{T}_{\mathrm{N}^{2}(\mathrm{t})}^{2}, \mathrm{t}\right]$. When $\mathrm{T}_{\mathrm{N}^{2}(\mathrm{t})}^{2}=\mathrm{t}$ then (3.19) is obvious. When $\mathrm{T}_{\mathrm{N}^{2}(\mathrm{t})}^{2}<\mathrm{t}$ then from (3.9) we have $\mathrm{T}_{\mathrm{N}^{2}(\mathrm{t})}^{2} \leq \mathrm{s} \leq \mathrm{t}<\mathrm{T}_{\mathrm{N}^{2}(\mathrm{t})+1}^{2}$ and from the above case, we obtain (3.19) in both cases. Then the formula (3.19) is completely proved.

To prove (3.14), firstly, we let:

$$
\begin{aligned}
& \mathrm{A}_{\mathrm{j}}(\mathrm{t}):=\left\{\exists \mathrm{s} \in<\mathrm{T}_{\mathrm{j}-1}^{2}, \mathrm{~T}_{\mathrm{j}}^{2}>: \mathrm{U}(\mathrm{s})<0\right\} \\
& \left(\forall \mathrm{j}=1 \div \mathrm{N}^{2}(\mathrm{t})+1\right) .
\end{aligned}
$$


In which (see (3.20)):

$$
\mathrm{A}_{1}(\mathrm{t}):=\left\{\exists \mathrm{s} \in\left(0, \mathrm{~T}_{1}^{2}\right): \mathrm{U}(\mathrm{s})<0\right\}=\phi .
$$

Then from (3.13) and (3.18), it is easy to see that:

$$
A(t)=\bigcup_{j=1}^{N^{2}(t)+1} A_{j}(t)=\bigcup_{j=2}^{N^{2}(t)+1} A_{j}(t)
$$

But from (3.19) and (3.21) we also find:

$$
\begin{aligned}
& \left\{\mathrm{U}\left(\mathrm{T}_{\mathrm{j}-1}^{2}\right)<0\right\} \subset \mathrm{A}_{\mathrm{j}}(\mathrm{t}) \subset\left\{\mathrm{U}\left(\mathrm{T}_{\mathrm{j}-1}^{2}\right)<0\right\} \\
& \Rightarrow \mathrm{A}_{\mathrm{j}}(\mathrm{t})=\left\{\mathrm{U}\left(\mathrm{T}_{\mathrm{j}-1}^{2}\right)<0, \forall \mathrm{j}=2 \div \mathrm{N}^{2}(\mathrm{t})+1\right\},
\end{aligned}
$$

On this basis (3.21) and (3.22) we get:

$$
A(t)=\bigcup_{j=1}^{N^{2}(t)+1} A_{j}(t)=\bigcup_{j=2}^{N^{2}(t)+1}\left\{U\left(T_{j-1}^{2}\right)<0\right\}=\bigcup_{j=1}^{N^{2}(t)}\left\{U\left(T_{j}^{2}\right)<0\right\},
$$

So, (3.14) is proven. When letting:

$$
\begin{aligned}
& \mathrm{B}_{\mathrm{j}}(\mathrm{t}):=\left\{\mathrm{U}\left(\mathrm{T}_{\mathrm{j}}^{2}\right)<0\right\} \\
& \Leftrightarrow \overline{\mathrm{B}_{\mathrm{j}}(\mathrm{t})}:=\left\{\mathrm{U}\left(\mathrm{T}_{\mathrm{j}}^{2}\right) \geq 0\right\}\left(\forall \mathrm{j}=1 \div \mathrm{N}^{2}(\mathrm{t})+1\right),
\end{aligned}
$$
infer:

We rely on (3.14) and the De Morgan duality rule to

$$
\overline{\mathrm{A}(\mathrm{t})}=\overline{\mathrm{B}(\mathrm{t})}=\bigcap_{\mathrm{j}=1}^{\mathrm{N}^{2}(\mathrm{t})} \overline{\mathrm{B}_{\mathrm{j}}(\mathrm{t})}=\left\{\mathrm{U}\left(\mathrm{T}_{\mathrm{j}}^{2}\right) \geq 0, \forall \mathrm{j}=1 \div \mathrm{N}^{2}(\mathrm{t})\right\}
$$

Therefore, in condition (3.15) event $\mathrm{A}(\mathrm{t})$ will not occur and conclusion number 1 . is completely proved.

To prove the rest, we rely on (3.4) and (3.5) to deduce the equivalence of the following events:

$$
\left\{\mathrm{N}^{2}(\mathrm{t})=0\right\}=\left\{\tau_{1}^{2}=\frac{-\ln \mathrm{v}_{1}^{2}}{\mu_{2}}>\mathrm{t}\right\}, \mathrm{v}_{1}^{2} \sim \mathrm{U}(0,1) .
$$

When the above event has occurred, from (3.8) and (3.13) we find that event $\mathrm{A}(\mathrm{t})$ will not happen and we get the conclusion number 2 .

Since random variables $\mathrm{U}\left(\mathrm{T}_{\mathrm{j}}^{2}\right)$ can be simulated by Lemma 3.2, so random event $\mathrm{A}(\mathrm{t})$ can also be simulated according to Lemma 3.3. Therefore, we can approximate the solution of the problem (2.10) in the following form:

$$
\psi(\mathrm{u}, \mathrm{t})=\mathrm{P}\{\mathrm{A}(\mathrm{t})\} \approx \frac{\mathrm{M}}{\mathrm{N}}
$$

Where $M$ is the number of occurrences of event $A(t)$ in $\mathrm{N}$ simulations and determined by the following algorithm:

\section{Algorithm 3.3.}

Input: initial capital $\mathrm{u}$, time $\mathrm{t}$, number of simulations $\mathrm{N}$, parameter $\mu_{1}$, parameter $\mu_{2}, G=\left\{x_{1}, x_{2}, \ldots, x_{n}, \ldots\right\}$;

$\mathrm{P}=\left[\mathrm{p}_{\mathrm{ij}}\right]_{\mathrm{n} \times \mathrm{n}}$ is transition matrix of $\left\{\mathrm{X}_{\mathrm{i}}\right\} ; \mathrm{Q}=\left[\mathrm{q}_{\mathrm{ij}}\right]_{\mathrm{n} \times \mathrm{n}}$ is transition matrix of $\left\{\mathrm{Y}_{\mathrm{i}}\right\}$

Output: Risk probability $\psi(\mathrm{u}, \mathrm{t})$
Comment: For the problem of determining the risk probability of this model, we only need to calculate and check the condition that capital receives negative values at the time of claim as in Lemma 3.2 and Lemma 3.3.

\section{Steps of the algorithm:}

Firstly, let $\mathrm{M}=0, \mathrm{~T}_{\mathrm{o}}^{2}=\mathrm{T}_{\mathrm{o}}^{1}=0, \mathrm{U}\left(\mathrm{T}_{\mathrm{o}}^{2}\right)=\mathrm{u}$

Step A. With each $\mathrm{j}=1,2, \ldots$ we perform the following steps:

A1. Simulate the time to claim $T_{j}^{2}$ (after the time of claiming $\mathrm{T}_{\mathrm{j}-1}^{2}$ in the previous time) by this formula: $\mathrm{T}_{\mathrm{j}}^{2}:=\mathrm{T}_{\mathrm{j}-1}^{2}-\frac{\ln \mathrm{v}_{\mathrm{j}}^{2}}{\mu_{2}}, \mathrm{v}_{\mathrm{j}}^{2} \sim \mathrm{U}(0,1)$, and check the inequality:

$$
\mathrm{T}_{1}^{2} \leq \mathrm{t}
$$

- If (3.23a) is false: terminate the $\mathrm{n}^{\text {th }}$ time simulation of event $\mathrm{A}(\mathrm{t})$.

If (3.23a) is true: simulate $Y_{j}$ depending on homogeneous Markov chain according to algorithm 3.1 and we move to step A2.

A2. Simulate the time to claim $\mathrm{T}_{\mathrm{i}}^{1}\left(\mathrm{i}=\mathrm{N}^{1}\left(\mathrm{~T}_{\mathrm{j}-1}^{2}\right)+1 \div \mathrm{N}^{1}\left(\mathrm{~T}_{\mathrm{j}}^{2}\right)\right.$ ) according to the iterative formula:

$$
\mathrm{T}_{\mathrm{i}}^{1}:=\mathrm{T}_{\mathrm{i}-1}^{1}-\frac{\ln \mathrm{v}_{\mathrm{i}}^{1}}{\mu_{1}}, \mathrm{~V}_{\mathrm{i}}^{1} \sim \mathrm{U}(0,1)
$$

Where $N^{1}\left(T_{j}^{2}\right)=N^{1}\left(T_{j-1}^{2}\right)$ when $\quad T_{N^{1}\left(T_{j-1}^{2}\right)+1}^{1}>T_{j}^{2}$. Otherwise, $\mathrm{N}^{1}\left(\mathrm{~T}_{\mathrm{j}}^{2}\right)$ is selected from the condition: $\mathrm{T}_{\mathrm{N}^{1}\left(\mathrm{~T}_{\mathrm{j}-1}^{2}\right)}^{1}<\mathrm{T}_{\mathrm{N}^{1}\left(\mathrm{~T}_{\mathrm{j}-1}^{2}\right)+1}^{1}<\ldots<\mathrm{T}_{\mathrm{N}^{1}\left(\mathrm{~T}_{\mathrm{j}}^{2}\right)}^{1} \leq \mathrm{T}_{\mathrm{j}}^{2}<\mathrm{T}_{\mathrm{N}^{1}\left(\mathrm{~T}_{\mathrm{j}}^{2}\right)+1}^{1}$.

A3. Simulate $\mathrm{X}_{\mathrm{i}}$ depending on homogeneous Markov $\begin{array}{llll}\text { chain according } & \text { to }\end{array}$ $\left(\mathrm{i}=\mathrm{N}^{1}\left(\mathrm{~T}_{\mathrm{j}-1}^{2}\right)+1 \div \mathrm{N}^{1}\left(\mathrm{~T}_{\mathrm{j}}^{2}\right)\right.$ ), so as to:

A4. Calculate $U\left(T_{j}^{2}\right)$ according to formula (3.6) and check inequality:

$$
\mathrm{U}\left(\mathrm{T}_{\mathrm{j}}^{2}\right) \geq 0
$$

If (3.23a) is true: Move back to step A1, with $\mathrm{j}:=\mathrm{j}+$ 1.

If $(3.23 \mathrm{~b})$ is false: terminate the $\mathrm{n}^{\text {th }}$ time simulation of event $\mathrm{A}(\mathrm{t})$ and assign $\mathrm{M}:=\mathrm{M}+1$.

Step B: After simulating $\mathrm{N}$ times event $\mathrm{B}(\mathrm{t})$ (repeat $\mathrm{N}$ times step A), approximately calculate the ruin probability: $\Psi(\mathrm{u}, \mathrm{t})=\frac{\mathrm{M}}{\mathrm{N}}$.

Notice 3.1. The aforementioned loop will stop with $\mathrm{j}=\mathrm{N}^{2}(\mathrm{t}): \mathrm{T}_{\mathrm{N}^{2}(\mathrm{t})}^{2} \leq \mathrm{t}<\mathrm{T}_{\mathrm{N}^{2}(\mathrm{t})+1}^{2}$. Then we finish the $\mathrm{n}^{\mathrm{th}}$ 
time simulation of event $A(t)$ (see (3.15)). In case $N^{2}(t)=$ 0 (see (3.16), the $\mathrm{n}^{\text {th }}$ time simulation of event $\mathrm{A}(\mathrm{t})$ will end immediately at step $\mathrm{A} 1$ with $\mathrm{j}=1$.

\section{Numerical Experiment Results}

\subsection{Simulation Ruin Probability of the Model (2.1)}

With input data: initial capital takes values: $\mathrm{u}=2 ; \mathrm{u}=3$; $\mathrm{u}=4 ; \mathrm{u}=5 ; \mathrm{u}=6 ; \mathrm{u}=7$; time $\mathrm{t}$ gets values: $\mathrm{t}=4, \mathrm{t}=6, \mathrm{t}$ $=10$; number of simulations $\mathrm{N}=1000$; interest rate $\mathrm{r}=$ 0,088 ; parameter $\mu=2,5$.

*The claim process $\left\{X_{n}\right\}$ follows a homogeneous Markov chain with transition matrix, $X_{n}$ are taken from $G$ $=\{0 ; 1 ; 2 ; 3 ; \ldots$.$\} .$

$$
P=\left[\begin{array}{cccc}
0,1 & 0,15 & 0,3 & 0,4 \\
0,1 & 0,15 & 0,4 & 0,5 \\
0,1 & 0,4 & 0,3 & 0,1 \\
0,1 & 0,2 & 0,4 & 0,5
\end{array}\right] ;
$$

We have compiled calculation software in Python environment to demonstrate algorithm 3.2, when running this program, we obtain simulation results of ruin probability for model (2.1) with hypothesis (4.1) given in table 4.1 below:

Table 4.1. Simulating the ruin probability of the model (2.1) with assumption (4.1)

\begin{tabular}{|c|c|c|c|c|c|}
\hline $\begin{array}{c}\text { Initial } \\
\text { capital }\end{array}$ & $\begin{array}{c}\text { Number of } \\
\text { simulations }\end{array}$ & $\begin{array}{c}\text { Interest } \\
\text { rate }\end{array}$ & \multicolumn{3}{|c|}{ Ruin Probability } \\
$\psi(\mathrm{u}, \mathrm{t})$ \\
\hline $\mathrm{u}$ & $\mathrm{N}$ & $\mathrm{r}$ & $\mathrm{t}=4$ & $\mathrm{t}=6$ & $\mathrm{t}=10$ \\
\hline 2 & 1000 & 0.08 & 0,5420 & 0,6150 & 0,6250 \\
\hline 3 & & & 0,3080 & 0,4120 & 0,5192 \\
\hline 4 & & & 0,1450 & 0,2412 & 0,3154 \\
\hline 5 & & & 0,0840 & 0,1502 & 0,2442 \\
\hline 6 & & & 0,0230 & 0,1152 & 0,1582 \\
\hline 7 & & & 0,0142 & 0,0421 & 0,1276 \\
\hline
\end{tabular}

\subsection{Simulation Ruin Probability of the Model (2.3)}

With input data: $\mathrm{u}=2 ; \mathrm{u}=3 ; \mathrm{u}=4 ; \mathrm{u}=5 ; \mathrm{u}=6 ; \mathrm{u}=7$; time $\mathrm{t}$ gets values: $\mathrm{t}=4, \mathrm{t}=6, \mathrm{t}=10$; number of simulations $\mathrm{N}=1000$; parameter $\mu_{1}=4$; parameter $\mu_{2}=2$;

*The premium process $\left\{X_{n}\right\}$ follows a homogeneous Markov chain with transition matrix, $X_{n}$ are taken from $G$ $=\{0 ; 1 ; 2 ; 3 ; \ldots$.$\} .$

$$
P=\left[\begin{array}{cccc}
0,1 & 0,15 & 0,3 & 0,4 \\
0,1 & 0,15 & 0,4 & 0,5 \\
0,1 & 0,4 & 0,3 & 0,1 \\
0,1 & 0,2 & 0,4 & 0,5
\end{array}\right]
$$

*The claim process $\{Y n\}$ follows a homogeneous Markov chain with transition matrix, Yn are taken from $\mathrm{G}$ $=\{0 ; 1 ; 2 ; 3 ; \ldots$.$\} .$

$$
\mathrm{P}=\left[\begin{array}{cccc}
0,1 & 0,1 & 0,25 & 0,4 \\
0,15 & 0,15 & 0,4 & 0,5 \\
0,1 & 0,35 & 0,3 & 0,2 \\
0,16 & 0,2 & 0,4 & 0,5
\end{array}\right] ;
$$

We have compiled calculation software in Python environment to demonstrate algorithm 3.3, when running this program, we obtain simulation results of ruin probability for model (2.3) with hypothesis (4.2) and (4.3) given in table 4.3 below:

Table 4.2. Simulating the ruin probability of the model (2.3) with assumptions (4.2) and (4.3)

\begin{tabular}{|c|c|c|c|}
\hline Initial capital & \multicolumn{3}{|c|}{$\begin{array}{c}\text { Ruin Probability } \\
\psi(\mathrm{u}, \mathrm{t})\end{array}$} \\
\hline $\mathrm{u}$ & $\mathrm{t}=4$ & $\mathrm{t}=6$ & $\mathrm{t}=10$ \\
\hline 2 & 0,034 & 0,086 & 0,185 \\
\hline 3 & 0,013 & 0,048 & 0,088 \\
\hline 4 & 0,011 & 0,034 & 0,064 \\
\hline 5 & 0,008 & 0,028 & 0,042 \\
\hline 6 & 0,006 & 0,013 & 0,026 \\
\hline 7 & 0,004 & 0,008 & 0,012 \\
\hline
\end{tabular}

\section{Conclusions}

This paper study two models: i)the claim amounts is an homogeneous Markov chain in classical model; ii) premiums amounts, claim amounts are homogeneous Markov chains in the general model does not have effects of interests.

The sequence of random variables in the article is considered as a series of Markov dependent random variables. The main results of this paper are Lemma 3.1, Lemma 3.2 and Lemma 3.3, which have built mathematical formulas for the simulation of the probability of insurance models considered in this article.

This paper has built the theoretical basis of simulation for (2.1) modes and (2.3) model. We have built algorithms 3.2 and 3.3 to simulate ruin probability for model (2.1) and model (2.3) with a series of regression dependent random variables. From the results of approximately calculating the ruin probability for model (2.1) given in table 4.1 and model (2.3) given in table 4.2 shows the conformity of the results of quantitative research with qualitative research, specifically:

When increasing the initial capital $u$ of insurance companies, the ruin probability will decrease. For each level of capital $u$, as time $t$ increases, the ruin probability will increase.

This study is a result of a research with the title 'Mathematical Models in Economics and Application in 
Solving Some Problems of Economics and the Social Sciences' led by Dr. Phung Duy Quang, Foreign Trade University, Vietnam.

\section{REFERENCES}

[1] Chunhao Cai, Weilin Xiao. "Simulation of an integro-differential equation and application in estimation of ruin probability with mixed fractional Brownian motion", Journal Integral Equations Applications, Vo.33, No.1, 1-17 (2021). DOI: 10.1216/jie.2021.33.1.

[2] David J. Santanaa, Luis Rincón. "Approximations of the ruin probability in a discrete time risk model", Modern Stochastics: Theory and Applications, Vol.7, No.3, 221-243 (2020) DOI: https://doi.org/10.15559/20-VMSTA158.
[3] H. U. Gerber. "Ruin theory in the linear model". Insurance: Mathematics and Economics, Vo.1, 177-184 (1982). DOI: https://doi.org/10.1016/0167-6687(82)90011-7.

[4] J. Grandell. Aspects of Risk Theory. Springer, New York, 1992.

[5] Phung Duy Quang, "Upper bounds for ruin probability in a generalized risk process under rates of interest with homogenous Markov chain claims and homogenous Markov chain premiums", Applied Mathematical Sciences, Vol.8, No.29, 1445-1454 (2014). DOI: http://dx.doi.org/10 .12988/ams.2014.4144.

[6] Phung Duy Quang, "Ruin Probability in a Generalised Risk Process under Rates of Interest with Homogenous Markov Chains", East Asian Journal on Applied Mathematics, Vol.4, No.3, 283 - 300 (2014). DOI: https://doi.org/10.4208/eaja m.051013.230614a. 